\title{
Quantum-chemical study of octafluoro-spirobi[triphosphazene]
}

\author{
Marian Gall ${ }^{1}$ and Martin Breza ${ }^{2}$ \\ ${ }^{1}$ Slovak Technical University, Intitute of Physical Chemistry and Chemical Physics \\ ${ }^{2}$ Slovak University of Technology in Bratislava
}

November 27, 2020

\begin{abstract}
The geometries of monocharged and neutral octafluoro-spirobi[triphosphazene] in singlet, doublet and/or triplet ground spin states were optimized. Their electronic structures are investigated in terms of Quantum Theory of Atoms-in-Molecules and compared with neutral hexafluorocyclotriphosphazene. The change of the total molecular charge implies mainly the change of the properties of the nitrogen atoms which are bonded to the central spiro-phosphorus atom. The charged systems in singlet spin states have stable structures of D2d symmetry only unlike the remaining ones of C2 symmetry within two geometry types. The existence of the less symmetric structures can be fully explained as a consequence of the (pseudo-) Jahn-Teller effect.
\end{abstract}

\section{Hosted file}

SpiroPhos10.pdf available at https://authorea.com/users/379201/articles/495486-quantumchemical-study-of-octafluoro-spirobi-triphosphazene 